Sir,

\section{Primary tuberculous keratoconjunctivitis}

Primary tuberculous infection of the ocular surface was not uncommon in the 19th and early 20th centuries but is now very rare.$^{1-6}$ There have been few reports of primary tuberculous lesions affecting both cornea and conjunctiva. ${ }^{7}$

\section{Case report}

A 13-year-old South African female patient presented with a 3-month history of an inflamed epibulbar mass lesion in her right eye, not responding to topical chloramphenicol treatment. The lesion was gradually enlarging and mildly painful. She had no significant medical history and was otherwise healthy. She had two close contacts in the community with pulmonary tuberculosis.

On examination, her visual acuity was $6 / 6$ bilaterally. A fleshy mass was situated at the superior limbus temporally, measuring $5 \mathrm{~mm} \times 5 \mathrm{~mm}$ and involving both cornea and conjunctiva (Figure 1). The lesion was raised, mobile, and nontender and displayed several engorged conjunctival 'feeder-vessels'. The anterior chamber showed no inflammatory activity and the posterior segment was normal. Her left eye was normal. She had no regional lymphadenopathy or skin lesions.

Clinically, the lesion resembled a juxtalimbal sessile conjunctival papilloma, with a differential diagnosis of conjunctival intraepithelial neoplasia and conjunctival squamous cell carcinoma with corneal invasion. A complete excision biopsy with localised lamellar keratectomy was therefore performed.

Histopathological analysis revealed granulomatous inflammation, areas of caseous necrosis (Figure 2), and positive staining for acid-fast bacilli.

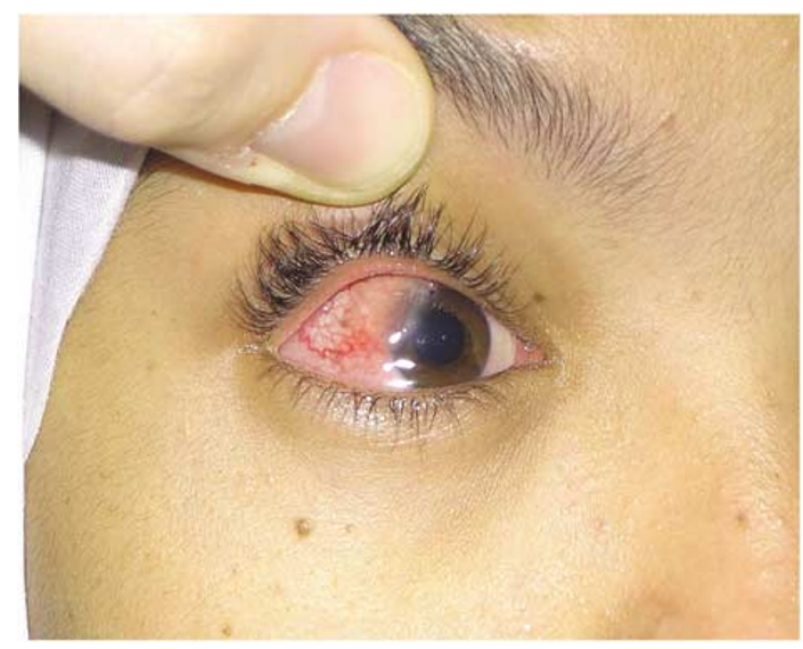

Figure 1 Tuberculous keratoconjunctivitis.

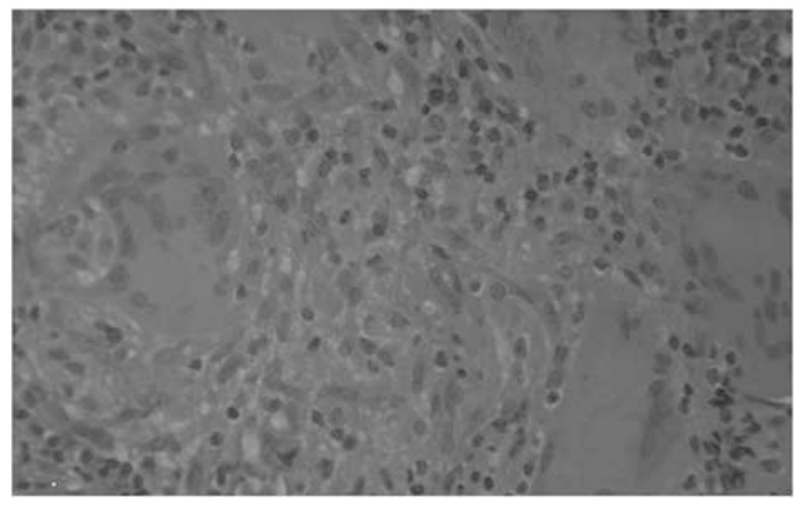

Figure 2 Granulomatous inflammation: giant cells and caseous necrosis.

Subsequent systemic examination and chest X-ray revealed no signs of extraocular tuberculosis. She was diagnosed with primary mycobacterium tuberculosis keratoconjunctivitis and started on 6 months of standard systemic antituberculous treatment of oral rifampicin, isoniazid, and pyrizanimide. ${ }^{4,7}$ The tuberculous lesion has shown no sign of recurrence.

\section{Comment}

Primary tuberculosis of the ocular surface occurs as a result of contamination from sputum droplets of patients with active respiratory tuberculosis causing an exogenous inoculation of bacilli. ${ }^{6,8,9}$ It has been found to be more common in the first two decades..$^{10}$ Lesions are typically unilateral and involve conjunctiva alone, often the palpebral conjunctiva. ${ }^{1,2}$ Conjunctival lesions can be described clinically as ulcerative, nodular, hypertrophic granulomatous, and pedunculated. ${ }^{10}$ Corneal lesions manifest as phlyctenulosis, infiltrates, ulcerations, and interstitial keratitis. ${ }^{11}$

Tuberculous keratoconjunctivitis is an uncommon presentation of ocular surface tuberculosis but should be considered in the differential diagnosis of atypical chronic ocular surface lesions, particularly in the developing world where the HIV pandemic has dramatically increased the incidence of pulmonary tuberculosis.

\section{References}

1 Samuelson A. Primary tuberculosis of the conjunctiva. Arch Ophthalmol 1936; 15: 975-984.

2 Archer D, Bird A. Primary tuberculosis of the conjunctiva. Br J Ophthalmol 1967; 51(10): 679-684.

3 Anhalt EF, Zavell S, Chang G, Byron HM. Conjunctival tuberculosis. Am J Ophthalmol 1960; 50: 265-269.

4 Cook CD, Hainsworth M. Tuberculosis of the conjunctiva in association with a neighbouring lupus vulgaris lesion. $\mathrm{Br} \mathrm{J}$ Ophthalmol 1990; 74(5): 315-316. 
5 Mckenzie WH. Tuberculosis of the conjunctiva. Report of a case. Am J Ophthalmol 1939; 22: 744-749.

6 Salas D, Murthy S, Champ C, Hawksworth N. Primary tuberculosis of the conjunctiva. Eye 2001; 15(Part 5): 674-676.

7 Cameron JA, Nasr AM, Chavis P. Epibulbar and ocular tuberculosis. Arch Ophthalmol 1996; 114(6): 770-771.

8 Flinnoff WC. Ocular tuberculosis, experimental and clinical. Arch Ophthalmol 1924; 53: 130-136.

9 Grunert C. A contribution to the subject of tuberculosis of the conjunctiva. Arch Ophthalmol 1899; 28: 540-556.

10 Eyre JWH. Tuberculosis of the conjunctiva: its etiology, pathology and diagnosis. Lancet 1912; 1: 1319-1328.

11 Aclimandos WA, Kerr-Muir M. Tuberculous keratoconjunctivitis. Br J Ophthalmol 1992; 76: 175-176.

\section{AG Zaborowski', BN Gundry', ME Masenya² and L Visser ${ }^{1}$}

${ }^{1}$ Department of Ophthalmology, Nelson R

Mandela School of Medicine, Durban,

South Africa

${ }^{2}$ Department of Anatomical Pathology, Nelson R Mandela School of Medicine, 52 Umbilo Road Congella, Durban, KwaZulu-Natal 4013, South Africa

Correspondence: A Zaborowski,

Tel: + 2731 3603450;

Fax: + 27315727634

E-mail: azaborowski@mweb.co.za

Eye (2006) 20, 978-979. doi:10.1038/sj.eye.6702090; published online 9 September 2005

Sir,

An atypical presentation of lattice corneal dystrophy in a patient with juvenile glaucoma

Lattice corneal dystrophy (LCD) is a bilateral, local amyloidosis, characterized by variation in the corneal manifestations, clinical course, and genetics. ${ }^{1}$ LCD has been attributed to TGFBI (transforming growth factor, beta-induced) gene on human chromosome 5q31 (OMIM No. 601692). LCD type II is associated with secondary open-angle glaucoma in the context of familial amyloidosis, Finnish (FAF). ${ }^{2}$ We report a case with the corneal findings of LCD type II but without any systemic involvement, in a patient with juvenile glaucoma.

\section{Case report}

A 26-year-old woman was referred to the glaucoma clinic because of elevated intraocular pressure (IOP) found in a routine eye examination. The patient was otherwise healthy with no significant family history, no visual complaints, or any history of recurrent erosions.

Her best-corrected visual acuity was 20/20 in both eyes. Corneal sensitivity was normal. Slit-lamp examination revealed refractile, radial filamentary lines in the anterior corneal stroma creating a lattice-work pattern, with sparing of the central cornea (Figure 1). No opacification or corneal haze was noted.

Gonioscopy showed high iris insertion and prominent iris processes in both eyes (Figure 2). IOP was $34 \mathrm{mmHg}$ in the right eye and $29 \mathrm{mmHg}$ in the left eye. Fundus examination revealed an arcuate nerve fibre loss between the first-order inferior temporal vessels of the left eye. Visual fields were normal. No change in the findings of the cornea, fundus, and visual fields was noted within the 5 years of follow-up. IOP remained controlled under antiglaucoma treatment.

Familial and systemic amyloidosis were ruled out by extensive physical examination and laboratory work-up. The patient did not present any skin lesions, macroglossia, arrhythmias or megalocardy, hepatosplenomegaly, arthritis, and cranial or peripheral neuropathy. There was no evidence of pulmonary, haematopoetic, or renal involvement. ESR, CRP, and immunoglobulins levels were within normal limits. Echocardiography was normal. Skin biopsy was negative

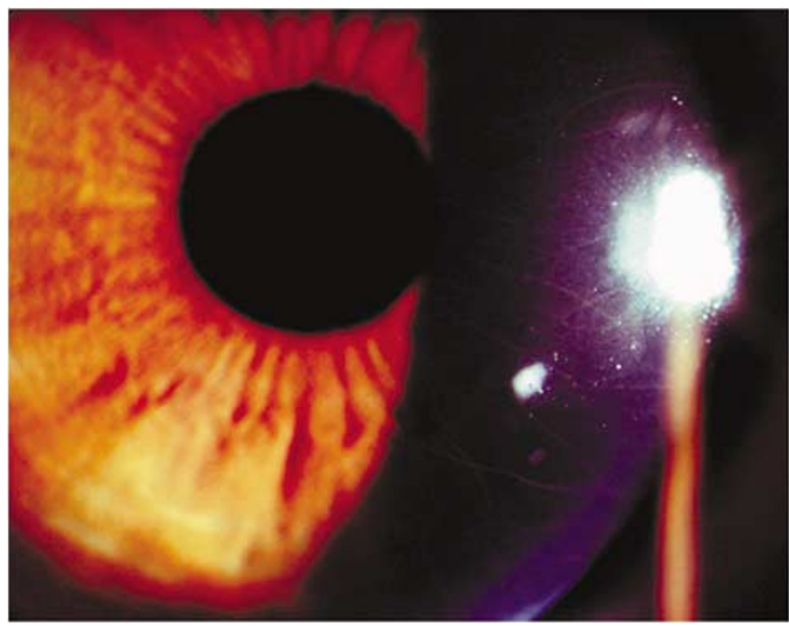

Figure 1 The patient's cornea is shown with refractile and discrete filamentary lines in the anterior stroma creating a lattice-work pattern. A white round dot in the anterior corneal stroma corresponding to an epithelial irregularity is remarkable. Note the absence of opacification, as well as the sparing of the central cornea. 